DOI $10.31489 / 2020$ No1/98-105

UDC: $62-531.7$

\title{
STUDY OF DYNAMIC PROCESSES IN BULK MATERIAL DURING ITS BAKING
}

\author{
Nizhegorodov A.I. ${ }^{1}$, Gavrilin A.N. ${ }^{2}$, Moyzes B.B. ${ }^{2}$, Kuvshinov K.A. ${ }^{2}$ \\ 1Irkutsk National Research Technical University, Irkutsk, Russia, nastromo_irkutsk@mail.ru \\ 2National Research Tomsk Polytechnic University, Tomsk, Russia, mbb@tpu.ru
}

The article presents the results of research on the dynamics of the base plate of an electric furnace for the heat treatment of bulk materials. The base plate with moving trolley on elastic bonds serves as the base which transports the heat-treated material in the hot part of the heating system with a specified speed and processing time. The experimental total elastic characteristic of the oscillating system is obtained; the amplitude-frequency characteristic is constructed, which characteristic is weak nonlinearity. Experiments have shown that conical springs of different designs do not provide a strong asymmetry in the base plate oscillations and therefore the vibrational transport efficiency is weak in the horizontal position of the prototype furnace. Further experiments were conducted when the furnace was installed at an angle to the horizontal surface. The vibrational transport time of vermiculite was reached 2.7...3.2 s, depending on the excitation frequency settings near the resonance zone. It is shown that the operation of the vibrating base plate may not be stable due to the high sensitivity of the excitation frequency from various factors. Methods for reducing the sensitivity coefficient are considered.

Keywords: bulk materials, electric furnace, vibrating base plate, oscillating system, nonlinear amplitudefrequency characteristic, oscillating system sensitivity to external factors.

\section{Introduction}

Electric furnaces with a vibration base platform [1] were developed as an alternative to the fire furnaces for vermiculite backing. First they operated on fuel oil and diesel fuel, and at present natural gas is used as a fuel. But their technical improvement stopped in the early 2000s, with the minimum energy intensity reached about $250 \ldots 260 \mathrm{~mJ} / \mathrm{m}^{3}$. Tests of new modern electric furnaces with a vibratory base platform showed that they more energy efficient and they significantly exceed the outdated fire furnaces: the specific energy intensity of vermiculite backing decreased to $75 \ldots 82$ $\mathrm{mJ} / \mathrm{m}^{3}$ [2]. This advantage is due to the fact that the energy of the fire torch is distributed in large working chambers with a volume of $1.2 \ldots 1.8 \mathrm{~m} 3$. With a capacity of $6 \mathrm{~m}^{3} /$ hour, the volume of the expanded material in it is $0.0062 \mathrm{~m}^{3}$, which is approximately $0.53 \%$ of the volume of the chamber. In electric furnaces of the same capacity, the heating energy is inclosed in a very small production space $(0.06 \ldots 0.08 \mathrm{~m} 3)$ between the base plate and the thermowell, where backing takes place. Here, the thermal field of electric heaters is incomparably more concentrated.

In addition to the high energy consumption of backing process, the fire furnaces have a number of serious drawbacks: air pollution by combustion products, high fire and explosion hazards, etc. new low energy consumption, furnaces accommodate more the requirements of environmental, industrial and fire safety [3-10]. The article "The development of baking technology for bulk materials based on the use of alternative electric furnace" reviewed the development of the design of an electric furnace prototype and conducted a static study of the parameters of an oscillating system. This research continues to study the dynamics of the developed system and the dynamics of bulk material. 


\section{Samples and Research Methods}

During the dynamic tests, there were calculated the peak values of the vibration displacement $(\mathrm{mm})$ and the root-mean-square vibration velocities $(\mathrm{mm} / \mathrm{s})$ and vibration accelerations $\left(\mathrm{m} / \mathrm{s}^{2}\right)$. The following measuring instruments and equipment were used: National instruments Compact DAQ9174 chassis with 4 slots for C-series modules, NI 9234 C-series input module and AP-85-100 vibration transducer. Figure 1 shows a mock-up model without a dispenser of heating system and thermal cover (photo), on which the experiments were conducted.

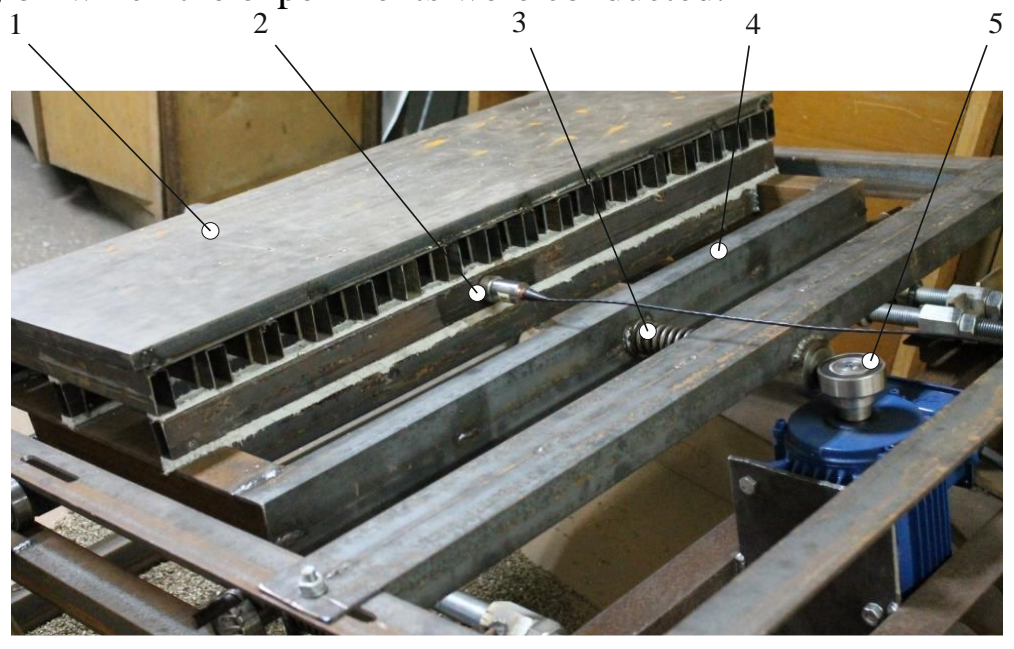

Fig.1. Physical model in the testing process:

1 - base plate, 2 - vibrator converter, 3 - pusher spring, 4 - moving trolley, 5 - excentric.

Table 1 shows the measurement results. The data from the first and second columns of the table were used to build the amplitude-frequency characteristic - the dependence of peak amplitude values on the excitation frequency $f(\mathrm{~Hz})$. Only the amplitude-frequency characteristic area near the resonance was investigated in order to evaluate the nonlinearity of the oscillating system of the experimental furnace at «large oscillations». A vibrodrive was started up (asynchronous engine 5AI $80 \mathrm{~V} 6 \mathrm{U} 2$ with nominal frequency $n=930 \mathrm{rpm}$ and $1.1 \mathrm{~kW}$ power), which excited vibrations of the moving trolley with the base plate and provided frequency control $f$. To control the oscillation frequency, the ACS 150 frequency converter was used.

Figure 2 shows the experimental graphs of the amplitude-frequency characteristic (AFC) of the moving trolley with the base plate on one-sided conical springs. The resonant point corresponds to the excitation frequency of $8.9 \mathrm{~Hz}$, which almost coincides with the calculated value of $8.77 \mathrm{~Hz}$. Graphs analysis shows that behavior of the oscillatory system does not fit into the classical picture characteristic of linear or nonlinear systems. Thus, when increasing the excitation frequency from 6.25 to $11.3 \mathrm{~Hz}$, the moving trolley with the base plate behaves like a nonlinear system with a socalled «soft» characteristic $[11,12]$. A specific feature of this effect is a jump-type transition from the pre-resonant mode of small oscillations to the post-resonant mode from point $a$ to point $b$, where the AFC is breaking [13-17]. However, in the opposite direction, when the excitation frequency decreases, the system goes through resonance at a peak amplitude value of $\sim 2.5 \mathrm{~mm}$ smoothly, without breaking the AFC, although its downward (left) branch is located almost vertically. The form of the obtained amplitude-frequency characteristic shows a very weak nonlinearity. A harmonic analysis of the oscillation of the moving trolley at resonance $x=f(t)$ detected the presence of a second harmonic with a frequency of $2 f$ and an amplitude of 12.4 times less than the amplitude of the main harmonic which corresponds to the excitation frequency. This means the presence of biharmonic oscillations, which can create an asymmetry of the oscillatory motion with a certain combination of harmonic phases and confirms the appearance of a weak nonlinearity of the system. But there was no noticeable asymmetry on the resonant oscillations oscillogram $(x)$. 
Table 1. The measurement results of oscillation parameters.

\begin{tabular}{|c|c|c|c|c|}
\hline No. & $\begin{array}{c}\text { Excitation } \\
\text { frequency, } \\
{[\mathrm{Hz}]}\end{array}$ & $\begin{array}{c}\text { Peak value of vibration } \\
\text { displacement amplitude, }[\mathrm{mm}]\end{array}$ & $\begin{array}{l}\text { Root-mean square value of } \\
\text { vibration velocity, }[\mathrm{mm} / \mathrm{s}]\end{array}$ & $\begin{array}{c}\text { Root-mean square value of } \\
\text { vibration acceleration, } \\
{[\mathrm{m} / \mathrm{s}]}\end{array}$ \\
\hline \multicolumn{5}{|c|}{ Excitation frequency increase, $f$} \\
\hline 1 & 6.25 & 0.06 & 1.7 & 0.177 \\
\hline 2 & 7.4 & 0.085 & 2.8 & 0.46 \\
\hline 3 & 7.6 & 0.08 & 2.6 & 0.428 \\
\hline 4 & 7.8 & 0.08 & 2.8 & 0.661 \\
\hline 5 & 7.9 & 0.082 & 2.9 & 0.51 \\
\hline 6 & 8.2 & 0.083 & 3.1 & 0.573 \\
\hline 7 & 8.4 & 0.086 & 3.2 & 0.588 \\
\hline 8 & 8.6 & 0.9 & 3.54 & 0.74 \\
\hline 9 & 8.7 & 0.1 & 3.87 & 0.796 \\
\hline 10 & 8.9 & 0.11 & 4.32 & 1.13 \\
\hline 11 & 9.1 & 1.98 & 79.8 & 4.82 \\
\hline 12 & 9.3 & 1.2 & 50.3 & 3.22 \\
\hline 13 & 9.5 & 0.77 & 32.3 & 2.57 \\
\hline 14 & 9.9 & 0.52 & 22.9 & 2.35 \\
\hline 15 & 10.5 & 0.33 & 15.4 & 2.01 \\
\hline 16 & 11.3 & 0.24 & 12.2 & 1.38 \\
\hline \multicolumn{5}{|c|}{ Excitation frequency reduction, $f$} \\
\hline 17 & 10.9 & 0.26 & 12.4 & 1.39 \\
\hline 18 & 10.1 & 0.45 & 20.6 & 1.57 \\
\hline 19 & 9.5 & 0.93 & 39.7 & 2.86 \\
\hline 20 & 9.1 & 1.5 & 62.6 & 3.67 \\
\hline 21 & 8.9 & 2.5 & 100.68 & 6.3 \\
\hline 22 & 8.9 & 2.5 & 104.6 & 6.3 \\
\hline 23 & 8.9 & 2.26 & 90.1 & 5.2 \\
\hline 24 & 8.7 & 0.59 & 23.09 & 1.75 \\
\hline 25 & 8.6 & 0.2 & 7.3 & 0.806 \\
\hline
\end{tabular}

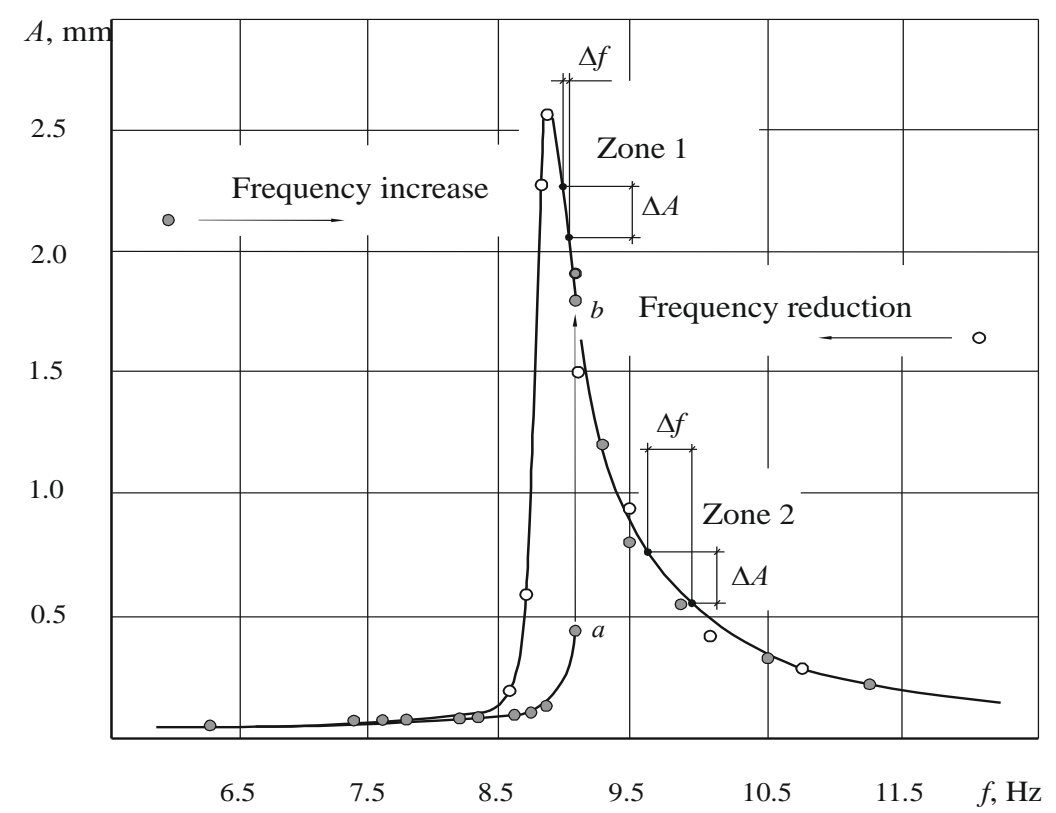

Fig.2. AFC of the oscillatory system. 
The exfoliated vermiculite was used when testing the vibrotransport effect in a horizontal position. There was no tendency to unidirectional movement, the chaotic grain movements were observed, which are unacceptable for reasons of wearability and breakage. The tip angle of the base plate is the only factor allows the unidirectional movement of the material on the platform. The increasing an angle to $9^{\circ}$ did not give an effect. The transport effect became discernable only at a tilt of $19^{\circ}$ and the post-resonant operation mode. The oscillatory system is extremely sensitive to the above-mentioned possible fluctuations due to the high quality factor, expressed in a narrow, elongated resonant peak of the AFC (Fig. 2). The ratio $\varepsilon=\Delta A / \Delta f$ estimates the sensitivity of the $\varepsilon$ system to the amplitude deviation during the fluctuation of the excitation frequency. The system is the most sensitive in zone 1 near resonance, since the slightest change in $\Delta f$, for example, $0.06 \mathrm{~Hz}$, leads to a change in the amplitude of oscillations by $0.22 \mathrm{~mm}$. The coefficient of sensitivity is approximately equal to $\varepsilon=3.7 \mathrm{~mm} / \mathrm{Hz}$. Whereas, in zone 2 at $\Delta f \approx 0.31 \mathrm{~Hz}$ and the same $\Delta A \approx 0.22$ $\mathrm{mm}$, the sensitivity coefficient is $\varepsilon=0.71 \mathrm{~mm} / \mathrm{Hz}$.

So, the sensitivity of the oscillatory system increases by $3.7 / 0.71=5.2$ when passing from zone 2 to zone 1 that is, it increases by more than five times. It is necessary to maintain steady vibration parameters near zone 2 (Figure 2) to ensure the stability of vibrotransporting of the flowing medium in the thermal field of the heating system of the furnace. Here, the feed rate is adjustable, but vibrational acceleration was insufficient $\left(1.38 \ldots 2.35 \mathrm{~m} / \mathrm{s}^{2}\right.$, table 1) for particles to overcome friction and start one-way traffic because of the type of conical springs installed on the moving trolley and due to weak non-linearity of the system when levels are horizontal.

The short conical springs with diameters at the top are $16 \mathrm{~mm}$ and at the base $64 \mathrm{~mm}$, with length $31 \mathrm{~mm}$ were tested to reinforce the nonlinearity of elasticity of the oscillatory system and to achieve the vibro-transport effect. They are designed the way that when fully compressed, the coils of the springs are fitted one into the other and the spring is compressed in length to the thickness of its coil. Figure 3 shows a fragment of the moving trolley with short conical springs and one of such springs.

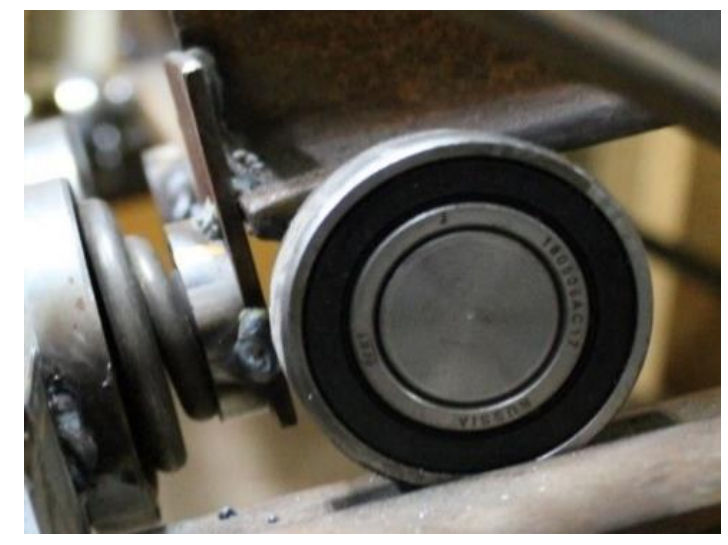

a

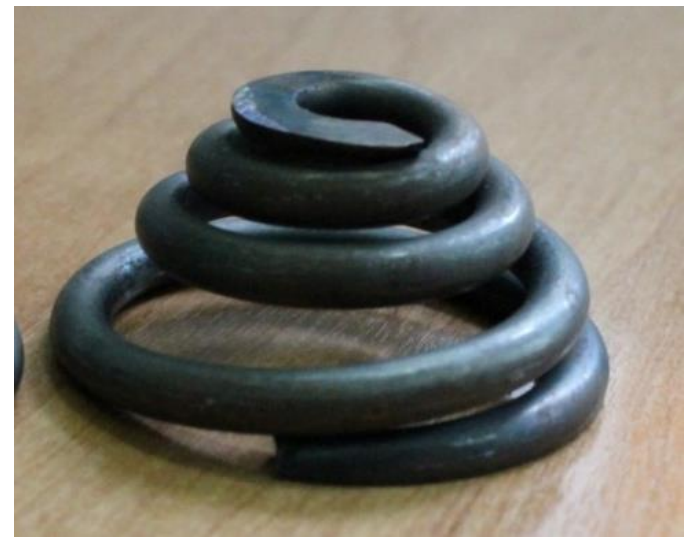

b

Fig.3. A fragment of the moving trolley with short conical springs $(a)$ and the spring itself $(b)$.

The total stiffness of the system decreased and became equal to $c=210031 \mathrm{~N} / \mathrm{m}$. The natural frequency calculated by the formula (1) in terms of $\mathrm{Hz}$, was $8.3 \mathrm{~Hz}$. During the experiments, the oscillation mode varied, but their frequency changed near the resonant peak of the oscillatory system, where the nonlinearity of the system is very strong. When it was located on the surface of the base plate, the expanded vermiculite (with grain size 2 to $8 \mathrm{~mm}$ ) tended to move to desired direction at the maximum amplitude (about $2.3 \mathrm{~mm}$ ) and in close to the resonance, when the furnace prototype was in horizontal position [18-21].

When the oven was installed at an angle of $9^{\circ}$, this tendency intensified, but the time for moving index marks made in the form of paper pellets and placed in a single-layer array of 
expanded vermiculite grains ranged from 15 to $20 \mathrm{~s}$ with a base plate length of $0.4 \mathrm{~m}$. Obviously, such movement mode could not provide the required performance of the furnace during the vermiculite baking $[22,23]$. For this it was necessary to achieve the time of movement of the material on the platform within 2.7...3.2 s. Therefore, the further of experiments were continued on the former conical springs at a tilt angle of $19^{\circ}$.

When measuring, the frame of the furnace prototype was set at an angle of $19^{\circ}$ (Figure 1). The cold exfoliated vermiculite was used for experiments. The bottom plate was also cold $\left(23 \ldots 24^{\circ} \mathrm{C}\right)$. The expanded vermiculite was poured on the base plate. It was leveled to obtain a single-layer array, evenly distributed over the surface without gaps between the grains. The index mark made in the form of paper pellet was put on the surface of the platform to track the movement of expanded vermiculite. The path section that index mark passed ranged from 0.39 to $0.3 \mathrm{~m}$. with the length of the base plate $l_{\mathrm{p}}=0.4 \mathrm{~m}$. The time was controlled by a stopwatch; and the average speed in each experiment at each relevant path section $l_{\Pi}$ was calculated using the formula:

$$
v=\frac{l_{\mathrm{p}}}{t} \text {. }
$$

The rotation frequency of the field in the stator of actuator coils of the driving motor $f_{\mathrm{m}}$ was controlled by digital indicator of the frequency converter and was recalculated to the oscillation frequency of the moving trolley $f$ according to the formula:

$$
f=\frac{930}{3000} \cdot f_{\mathrm{m}}
$$

where 3000 - AC mains frequency in $\mathrm{rpm}(50 \mathrm{~Hz}), 930$ - engine revolutions in rpm.

Since in each experiment the path sections were different, the average movement time of vermiculite grains through the full length of the base platform was calculated using the formula:

$$
t_{\mathrm{m}}=\frac{l_{\mathrm{p}}}{v_{\mathrm{m}}} .
$$

and the average speed in three experiments was determined as an arithmetic average value.

There are the results of one of the experiments in table 2 as an example.

Table 2. The speed and time of vermiculite movement at $f=9.13 \mathrm{~Hz}$

\begin{tabular}{|c|c|c|c|c|}
\hline $\begin{array}{c}\text { Path section, } l, \\
{[\mathrm{~m}]}\end{array}$ & $\begin{array}{c}\text { Movement time, } t, \\
{[\mathrm{~s}]}\end{array}$ & $\begin{array}{c}\text { Speed, } v, \\
{[\mathrm{~m} / \mathrm{s}]}\end{array}$ & $\begin{array}{c}\text { Average speed, } v_{\mathrm{m}}, \\
{[\mathrm{m} / \mathrm{s}]}\end{array}$ & $\begin{array}{c}\text { Average time, } \\
t_{\mathrm{m}},[\mathrm{s}]\end{array}$ \\
\hline 0.39 & 2.47 & 0.158 & - & - \\
\hline 0.37 & 2.46 & 0.15 & - & - \\
\hline 0.37 & 3.07 & 0.121 & - & - \\
\hline- & - & - & 0.143 & 2.79 \\
\hline
\end{tabular}

Figure 4 shows the dependence of the average movement time of index marks on the base plate surface on oscillations frequency in a narrow post-resonant range. In the area from 8.9 to $9.1 \mathrm{~Hz}$ (at the very beginning of post-resonant zone, at a resonance of $8.9 \mathrm{~Hz}$ ), the movement time does not change. The amplitude decrease in this range is compensated by an increase in the angular frequency $\omega$ from 55.9 to $57.1 \mathrm{rad} / \mathrm{s}$. Therefore, the vibration acceleration determined by the formula $\left(\mathrm{m} / \mathrm{s}^{2}\right)$ :

$\ddot{x}=A \omega^{2}$,

under the condition of harmonic oscillations, also does not change.

However, a further increase in the oscillation frequency leads to an increase in the vibration acceleration and the movement time of vermiculite grains on the base platform and, approximately at a frequency of $9.13 \mathrm{~Hz}$, the time of movement becomes equal to $2.8 \mathrm{~s}$, which corresponds to the average baking time of vermiculite concentrates. For this oscillatory system figure 4 shows the 
frequency range $(9.12 \ldots 9.16 \mathrm{~Hz})$ where the baking time is controlled depending on the type and size of the heat-treating material.

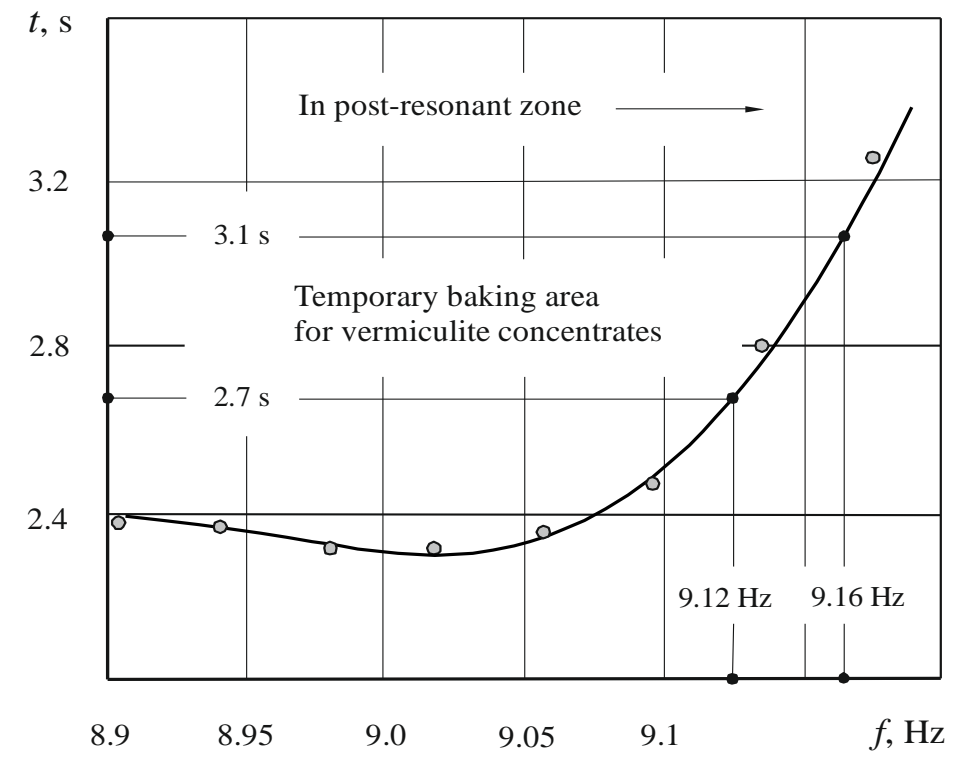

Fig.4. The time change of index mark movement in single-layer array of vermiculite in post-resonant zone.

\section{Results and discussion}

Thus a prototype furnace with a vibratory base plate was tested and experiments proved that this design can be used for building an industrial three-module furnace for baking vermiculite and other potentially high-temperature-activated bulk materials, for example, sungulite-vermiculite [2226]. At the same time, its efficiency will be significantly higher than the known modular trigger furnaces by reducing the baking energy intensity from $170 \ldots 175$ to $75 \ldots 82 \mathrm{~mJ} / \mathrm{m}^{3}$.

The vibratory base plate on the moving trolley is a vibrating dispenser which provides the flow and time-controlled movement of bulk materials in the thermal field of the electric heating system of the furnace due to the vibrating effect. Experiments have shown that the purpose of research has been achieved. A rational design of the furnace oscillating system has been obtained, the modes of vibratory displacement of bulk materials have been worked out, the frequency range in which heat treatment is carried out for a duration of 2.7...3.1 s has been determined.

\section{Conclusion}

Analyzing the obtained results, it is necessary to consider that some shortcomings require further work on the improvement of such furnaces. It has already been noted above that due to the high quality factor, the oscillating system is too sensitive to the above-mentioned possible fluctuations in frequency, rigidity, friction and other factors in high-temperature conditions and the instability of the electrical network. For example, the oscillatory system sensitivity to fluctuations of the excitation frequency in different zones of the frequency response may vary by more than five times, and this will affect the heat treatment time.

Therefore, it is necessary to achieve the possibility of vibro-transporting of bulk medium at horizontal position of the base plate due to non-symmetrical vibrations. It is necessary to create a strong nonlinear elastic behavior of the system, so that its resonance peak would be strongly "bend" to the left $[11,17]$, and then the sensitivity coefficient of amplitude $\varepsilon$ to fluctuations of the excitation frequency can be significantly reduced.

When tilted, the round particles of some bulk materials can simply roll under the gravity, not due to the vibro-transport effect, which will reduce their processing time. There is another important 
reason for horizontal installation of the base plate. If there is a slope, an air draft effect occurs, which will cause the movement of external air in the baking space under the thermal cover of the module. It will lead to cooling of the heating system of the furnace unit.

The relative error of the frequencies of the resonant point is only:

$$
\Delta=\frac{8.90-8.77}{8.90} \cdot 100 \%=1.46 \% \text {. }
$$

This allows us to conclude about the validity of the results. The research is a continued of the previous authors studies [1-3]. The obtained results allow us to continue work in the development of the electric furnace for the heat treatment of bulk materials. The novelty of the approach used in this study is confirmed by a number of patents for inventions mentioned in the research [1].

\section{Acknowledgements}

The research is carried out at Tomsk Polytechnic University within the framework of Tomsk Polytechnic University Competitiveness Enhancement Program grant.

\section{REFERENCES}

1 Nizhegorodov A.I. Energy efficient electric furnace with moving hearth platform for firing vermiculite. Refractories and Industrial Ceramics. 2017, Vol. 58, No. 1, pp. 29 - 34. doi: 10.1007/s11148 017-0049-4.

2 Nizhegorodov A.I., Bryanskikh T.B., Gavrilin A.N., Moyzes B.B., Vavilova G.V., Gradoboev A.V., Tlusty J., Tuzikova V. Testing a new alternative electric furnace for vermiculite concentrates heat treatment. Bulletin of the Tomsk Polytechnic University, Geo Assets Engineering. 2018, Vol. 329, No 4, pp. $142-153$.

3 Nizhegorodov A.I. The effectiveness of fire and electrical module and trigger kilns vermiculite. Engineering technology. 2010, Vol. 1, pp. $32-34$.

4 Lozano-Lunar A.at el. Safe use of electric arc furnace dust as secondary raw material in selfcompacting mortars production. J. of Cleaner Production. 2019, Vol. 211, pp. 1375 - 1388.

5 Santamaria A., Faleschini F., Giacomello G., et al. Dimensional stability of electric arc furnace slag in civil engineering applications. J. of Cleaner Production. 2018, Vol. 205, pp. $599-609$.

$6 \mathrm{Xu} \mathrm{W.,} \mathrm{Zhang} \mathrm{J.,} \mathrm{Zhang} \mathrm{R.} \mathrm{Application} \mathrm{of} \mathrm{multi-model} \mathrm{switching} \mathrm{predictive} \mathrm{functional} \mathrm{control} \mathrm{on}$ the temperature system of an electric heating furnace. ISA Transaction. 2017, Vol. 68, pp. $287-292$.

7 Bryanskikh T.V., Kokourov D.V. Energy efficiency of electric furnaces with movable floor in firing of vermiculite concentrates of different size groups. Refractories and Industrial Ceramics. 2017, Vol. 58, pp.368 - 373. doi: 10.1007/s11148-017-0113-0.

8 Zvezdin A.V., Bryanskikh T.B. Considering adaptation of electrical ovens with unit-type releasing to peculiarities of thermal energization of mineral raw materials. IOP Conf. Series: Materials Science and Engineering. 2017, Vol. 168, No.1, pp. 012003.

9 Bryanskikh T.V. A study of the energy efficiency of electric furnaces with vibrating movable platform for heat treatment of mineral ore. Vest. IrGTU. 2016, Vol. 12, pp. $12-19$.

10 Tymchak V.M., Gusovskii V.L. Design of heating and thermal furnaces. Reference. Moscow, Metallurgiya, 1983, 480 p. [in Russian]

11 Blekhman I.I. Vibrations in engineering: reference book. Oscillations of nonlinear mechanical systems. Moscow, Mashinostroenie, 1979, 351 p. [in Russian]

12 Bauman V.A., Bykhovskij I.I. Vibration machines and processes in construction. Moscow, Vysshaya shkola, 1977, 255 p. [in Russian]

13 Gvozdkova S.I., Shvartsburg L.E. Analysis of sources and methods for reducing noise by minimizing vibrations of engineering technological processes. Procedia Engineering. 2017, Vol. 206, pp.958 - 964.

14 Mo J.P.T., Cheung S.C.P., Das R. Demystifying Numerical Models. Oxford, 2019, 274 p.

15 Sofiyev A.H. Review of research on the vibration and buckling of the FGM conical shells. Composite Structures. 2019, Vol. 211, pp. $301-317$. 
16 İşçi S. Intercalation of vermiculite in presence of surfactants. Applied Clay Science. 2017, Vol. 146, pp. $7-13$.

17 Ritz M., Zdrálková J., Valášková M., Vibrational spectroscopy of acid treated vermiculites. Vibrational Spectroscopy. 2014, Vol. 70, pp. 63-69.

18 Surzhikov A.P., Frangulyan T.S., Ghyngazov S.A. A dilatometric study of the effect of pressing on the kinetics of compression of ultrafine zirconium doxide powders under thermal annealing. Russ. Phys. J. 2012, Vol.55, No.4, pp. 345 - 352. doi: 10.1007/s11182-012-9818-1.

19 Surzhikova O. Power supply of remote and almost inaccessible settlements. IOP Conference Series: Materials Science and Engineering IOP. 2015, Vol. 81, No.1, pp. 012098. doi 10.1088/1757899X/81/1/012098

20 Bemš, J., Starý, O., Macaš, M., Žegklitz, J., Pošík, P. Innovative default prediction approach. Expert Systems with Applications. 2015, Vol. 42, No. 17-18, pp. 6277 - 6285 . doi: 10.1016/j.eswa.2015.04.053.

21 Surzhikov A.P., Peshev V.V., Pritulov A.M., Gyngazov, S.A. Grain-boundary diffusion of oxygen in polycrystalline ferrites. Russian Physics Journal, 1999, Vol.42, No.5, pp. 490 - 495. doi: 10.1007/ BF02508222.

22 Kremenetskaya I.P., et al. Serpentine minerals amorphization in the technology of magnesiasilicate reagent for immobilization of heavy metals. Chemistry for Sustainable Development. 2010, Vol.18, pp. $41-49$.

23 Viti C., Hirose T. Thermal decomposition of serpentine during coseismic faulting: Nanostructures and mineral reactions. J. of Structural Geology. 2010, Vol. 32, No.10, pp. $1476-1484$.

24 Li J., Hitch M. Mechanical activation of magnesium silicates for mineral carbonation, a review. Minerals Engineering. 2018, Vol. 128, pp. $69-83$.

25 Plotnikova I., Redko L., Titova O., Chaykovskaya O. Development of the Production Process Model Based on the Controllable Input Factors. IOP Conf. Series: Materials Science and Engineering, 2016, Vol.132, pp. 012023. doi.org/10.1088/1757-899X/132/1/012023.

26 Redko V.V., Starikova N.S., Redko L.A., Vavilova G.V. Determination of sensitivity for inprocess control of cable product insulation. IOP Conf. Series: Materials Science and Engineering. 2015, Vol. 81, No.1, pp. 012083. DOI: 10.1088/1757-899X/81/1/012083. 